\title{
SEGMENTATION OF BRAIN IMAGES BY OPTIMIZING CLUSTERING OF CONVOLUTION BASED FEATURES
}

\author{
Vikas Kumar \\ E-Mail:-chawla.vikas1@gmail.com \\ Galaxy Global Group Of Institutions \\ Ambala-Haryana, INDIA \\ Affiliated to Kurukshetra University,Kurukshetra
}

\begin{abstract}
Brain tumour segmentation aims to separate the various types of tumour tissues like active cells, necrotic core, and edema from normal brain tissues of substantia alba (WM), grey matter (GM), and spinal fluid (CSF). Magnetic Resonance Imaging based brain tumour segmentation studies are attracting more and more attention in recent years thanks to non-invasive imaging and good soft tissue contrast of resonance Imaging (MRI) images. With the event of just about two decades, the ingenious approaches applying computer-aided techniques for segmenting brain tumour are getting more and more mature and coming closer to routine clinical applications. the aim of this paper is to supply a comprehensive overview for MRIbased brain tumour segmentation methods. Firstly, a quick introduction to brain tumours and imaging modalities of brain tumours is given in this proposed research, convolution based optimization. These stepwise step refine the segmentation and improve the classification parameter with the assistance of particle swarmoptimization.
\end{abstract}

Keywords: Magnetic Resonance Imaging, Spinal fluid, Grey Matter, Substantia alba, CNNs, DNN .

\section{INTRODUCTION}

Brain region segmentation or skull stripping is an important step in neuroimaging applications like surgical, surface reconstruction, image registration, etc. [1] [2]. The accuracy of all present methods depends on the registration and geometry of the image. When this fails, the probability of success is extremely less. To avoid this, Convolutional Neural Network $(\mathrm{CNN})$ is employed for brain extraction which is free from geometry and registration. It learned the connectedness and shape of the brain. Accurate diagnosis in the procedure has attained using different imaging modalities like Magnetic Resonance(MR) imaging, computerized tomography (CT), digital mammography, etc [7] [9]. These can provide very detailed and informative anatomy of a topic. The research community develops many methods. Deep learning, called deep structured learning is one of the machine learning algorithms. It learns data from the input image using either supervised or unsupervised [3] [12]. There has been a big effort in developing classical machine learning algorithms for the segmentation of normal (e.g., substantia alba and grey matter) and abnormal brain tissues (e.g. brain tumours). However, the creation of the imaging features that enable such segmentation requires careful engineering and specific expertise. Furthermore, traditional machine learning algorithms don't generalize well [13] [14]. Although major researches has been done by the medical imaging researcher society, automatic segmentation of the brain structures and detection of the abnormalities remain an unsolved problem. This is due to normal anatomical variations in brain morphology, variations in acquisition settings, variations within the appearance of pathology, MRI scanners and image acquisition imperfections. An emerging machine learning technique mentioned as deep learning can help avoid limitations of classical machine learning algorithms, and its self-learning of features may enable identification of the latest useful imaging features for quantitative chemical analysis of the brain [11]. Deep learning techniques are gaining popularity in many areas of medical image analysis like computer-aided detection of breast lesions, computer-aided diagnosis of breast lesions and pulmonary nodules, and in histopathological diagnosis.

\subsection{Brain Region Segmentation}

Brain tissue classification or segmentation is employed for the detection and diagnosis of normal and pathological tissues like MS tissue abnormalities and tumours. These abnormalities might be identified by tracking changes in volume, shape, and regional distribution of brain tissue during the follow-up of patients. Medical image segmentation is an important step for many subsequent image analysis tasks. The segmentation of anatomic structure within the brain plays an important role in Nero imaging analysis. Successful numerical algorithms can help researchers, physicians and neurosurgeons to research and diagnose the structure and performance of the brain in both health and disease. This has motivated the necessity for segmentation techniques that are robust in an application involving abroad range of anatomic structure, disease, and image type. the method of partitioning a digital image into multiple regions or sets of pixels is named image segmentation. Partitions are different objects in an image that have an equivalent texture or color. The results of image segmentation may be a set of regions that collectively cover the whole image or a group of contours extracted from the image[2][5].

\subsection{Brain Segmentation Approaches}

The details of Approaches as follows:

A. Edge-based Technique for Brain Image Segmentation: Edge detection techniques transform images to edge images 
taking advantage of the changes of grey tones within the images. The Advantageous result of this is that, edge image is obtained without encountering any changes in the physical characteristics of the most image.

(a) ACO Approach: The $\mathrm{ACO}$ (Ant Colony Optimization) based image edge detection approach aims to utilize a variety of ants to maneuver on a 2-D image for constructing a pheromone matrix, which represents the string information at each pixel location of the image [10]. ACO scheme starts from the initialization process, then runs for $\mathrm{N}$ number of times to construct the pheromone matrix by iteratively performing both the development process and therefore the update process. Finally, the choice process is performed to work out the sting.

(b) Symbolic logic Approach: Cristiano Jacques Miosso and Adolfo Bauchspiess evaluated the performance of a fuzzy inference system (FIS) in edge detection. It had been inferred that even though the advanced computational effort in comparison to the Sobel operator, the FIS(Fuzzy Inference System) system presents greater robustness to contrast and lighting variations, besides avoiding obtaining double edges.

(c) Neural Network Approach: Many image-based edge detection algorithms are using neural networks, the most successful system was introduced by Rowley et. al. The neural network technique in this section is such an approach and functions just like a pattern classifier, which collects the input features and outputs the decisions.

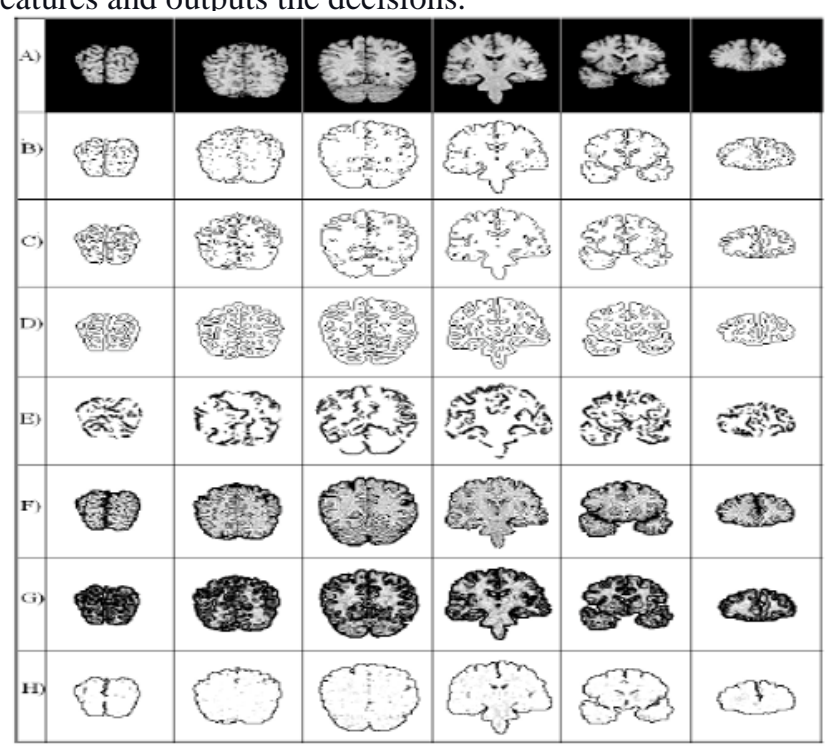

Fig 1.1: Edge based technique for brain image segmentation, a) Original images, b) using Prewitt method, c) using Roberts method, d) using Sobel method, e) using ACO method, f) using Fuzzy logic, g) using GA, h) using Neural Network [3][15] (d) GA Approach: GAs are robust therein they're not suffering from spurious local optima within the solution space. This robustness is protected by a robust mathematical foundation. The most interesting genetic application in edge detection is given by Gudmundsson and is as described below. Edges are represented during a binary image, where each pixel takes on either the worth zero (off) for a non-edge pixel or one (on) for a foothold pixel. Each pixel within the binary map corresponds to an underlying pixel within the original image. This edge representation is straightforward, allows direct illustration of results, location of edge points maps directly onto the first image and, adjacency and orientation are preserved. By using the sting map as an answer space for the GA, no special mappings are required, small neighbourhood windows are often overlaid, and edge structures and pixels are often modified on an area, intuitive basis.

\section{B. Splite and Merge Technique for Brain Image Segmentation:}

One of the basic properties of segmentation is the existence of a predicate which measures the region homogeneity. If this predicate is not satisfied for some region. On the other hand, if the predicate is satisfied for the union of two adjacent regions,

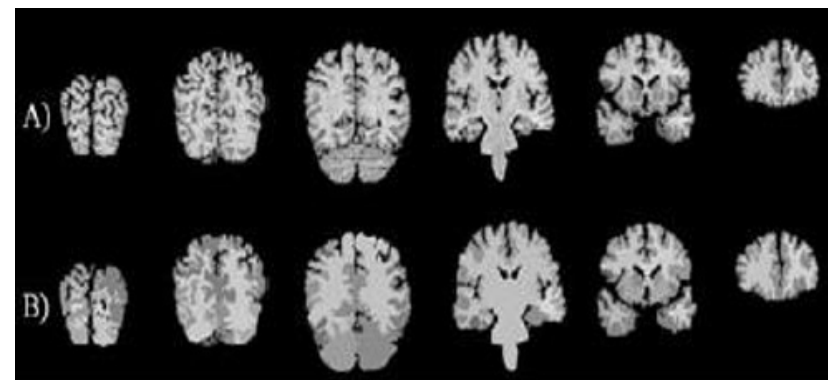

Fig. 1.2: Using Splite and Merge algorithm for brain image segmentation[16]

then these regions are collectively homogeneous and should be merged into a single region. A method towards the satisfaction of these homogeneity criteria is the split-andmerge algorithm [4] [5]. Figure 2 shows the example image using splite and merge algorithm for brain image segmentation.

C. Hybrid Method for Brain Image Segmentation: This Method is using granular rough sets for brain image segmentation. Recently, rough set theory has become a popular mathematical framework for granular computing and is used as a mathematical tool to analyze vagueness and uncertainty inherent in making decisions. The focus of the rough set theory is on the ambiguity caused by the limited discernibility of objects in the domain of discourse.

\subsection{Deep Learning}

Deep learning refers to neural networks with many layers (usually more than five) that extract a hierarchy of features 
from raw input images. It is a new and popular type of machine learning techniques that extract a complex hierarchy of features from images due to their self-learning ability as opposed to the hand-crafted feature extraction in classical machine learning algorithms. They achieve impressive results and generalizability by training on a large amount of data.

This allowed the training of deep learning algorithms with millions of images and provided robustness to variations in images. Some of the known deep learning algorithms are stacked auto-encoders, deep Boltzmann machines, deep neural networks, and convolutional neural networks (CNNs). CNNs are the most commonly applied to image segmentation and classification. CNNs were first introduced in 1989 [11], but gained great interest after deep CNNs [1] achieved spectacular results in Image in 2012. A typical CNN architecture contains subsequent layers of convolution, pooling, activation, and classification (fully connected). The convolutional layer produces feature maps by convolving a kernel across the input image. The pooling layer is used to down sample the output of preceding convolutional layers by using the maximum or average of the defined neighbourhood as the value passed to the next layer. Rectified Linear Unit (ReLU) and its

modifications such as Leaky ReLU are among the most commonly used activation functions. ReLU nonlinearly transforms data by clipping any negative input values to zero while positive input values are passed as output [4].

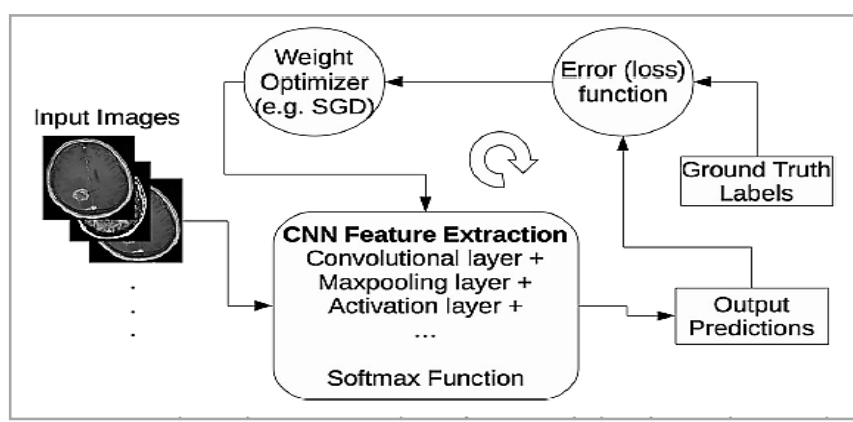

Fig. 1.3: A schematic representation of a convolutional neural network $(\mathrm{CNN})$ training process

\subsection{Brain Region Segmentation using CNN}

A fully automated system for brain region segmentation by using a Human intelligence-based deep learning technique is proposed. The deep learning technique is the most popular state of the art method in recent applications. Figure. 1.4 shows the flow diagram of the proposed methodology. There are two stages in this process: pre-processing and segmentation via Convolutional Neural Network (CNN). The noisy MRI image is used as an input image. MRI images are collected from a publicly available database Open Access Series of Image Studies (OASIS), to segment the brain region three-layered approach is used in this network.
A. Pre-processing: The MRI images are first undergoes to pre-processing step to enhance the quality of image for segmentation. In this work, Non Local Mean Filter is used for image de-noising which calculates weighted average of pixels and finding similarity with the target pixel. It consists of four steps.

Step 1: For the data redundancy among the "patches" of the noisy image the weighted average non-local pixel is used, and the noise free pixel is restored. The restored intensity, $\mathrm{NL}(\mathrm{u})\left(\mathrm{x}_{\mathrm{i}}\right)$ of the noisy pixel $\mathrm{u}\left(\mathrm{x}_{\mathrm{j}}\right)$ in the search window $V_{\mathrm{i}}$ is given by:

$$
\mathrm{NL}(\mathrm{u})\left(\mathrm{x}_{\mathrm{j}}\right)=\sum_{\mathrm{x}_{\mathrm{j}} \in \mathrm{v}_{\mathrm{j}}} \mathrm{w}\left(\mathrm{x}_{\mathrm{i}}, \mathrm{x}_{\mathrm{j}}\right) \mathrm{u}\left(\mathrm{x}_{\mathrm{j}}\right)
$$

Where, $M$ is the radius of the search window $V_{i}\left(x_{i}, x_{j}\right)$ is the weight allocate to the noisy value $u\left(x_{j}\right)$ to establish the intensity at $\mathrm{u}\left(\mathrm{x}_{\mathrm{i}}\right)$ voxel $\mathrm{x}_{\mathrm{i}}$.

Step 2: The weight is a measure of similarity between the intensity of the two locale patches $\mathrm{Ni}$ and $\mathrm{Nj}$ concentrate on voxels $\mathrm{X}_{\mathrm{i}}$ and $\mathrm{X}_{\mathrm{j}}$ is estimated by the weight such that $w\left(x_{i}, x_{j}\right) \in[0,1]$

Step 3: the squared Euclidean distance between intensity patches $\mathrm{u}\left(\mathrm{N}_{\mathrm{i}}\right)$ and $\mathrm{u}\left(\mathrm{N}_{\mathrm{j}}\right)$ based weight is given as:

$$
\mathrm{w}\left(\mathrm{x}_{\mathrm{i}}, \mathrm{x}_{\mathrm{j}}\right)=\frac{1}{2} \exp \left(-\frac{\left\|\mathrm{u}\left(\mathrm{N}_{\mathrm{i}}\right)-\mathrm{u}\left(\mathrm{N}_{\mathrm{j}}\right)\right\| \frac{1}{2}}{\mathrm{~h}^{2}}\right)
$$

Where, $\Sigma_{\mathrm{x}_{\mathrm{j}} \in v_{\mathrm{j}}} \mathrm{w}\left(\mathrm{x}_{\mathrm{i}}, \mathrm{x}_{\mathrm{j}}\right)=1$ is ensured by the normalization constant, $\mathrm{Zi}$ is the variable for exponential decay control, $\mathrm{h}$ is given by, $h=k \sigma$ where $\mathrm{k}$ is the smoothing parameter and $\sigma$ is the noise standard deviation. The noise is greatly reduced by using Non Local Mean filter algorithm.

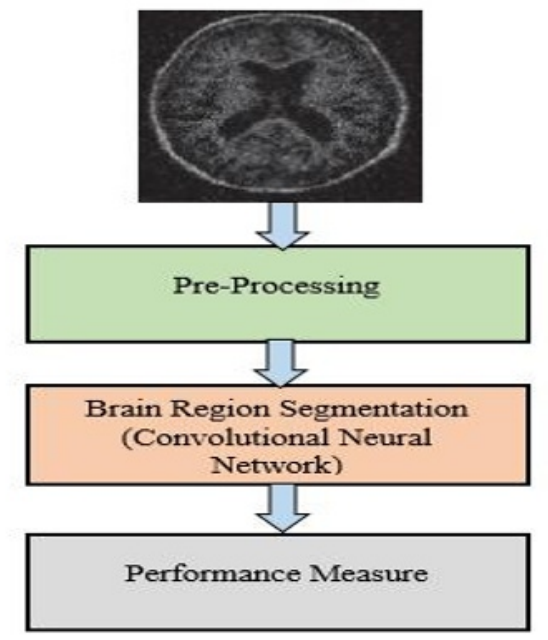

Fig. 1.4: Flow Diagram of the used methodology

It is an effective method to reduce the noise and it takes less time. One of the advantages of using Non Local Mean (NLM) filter is it does not loss any data or information related to the input image.

To perform a prediction of an input data, the output scores of the final CNN layer are connected to loss function (e.g., cross- 
entropy loss that normalizes scores into multinomial distribution over labels). Finally, parameters of the network are found by minimizing a loss function between prediction and ground truth labels with regularization constraints, and the network weights are updated a teach iteration (e.g., using stochastic gradient descent-SGD)using back propagation until convergence as shown in figure 1.3.

\subsection{CNN Architecture Styles}

1. Patch-Wise CNN Architecture: This is a simple well known approach to train a $\mathrm{CNN}$ algorithm for segmentation. An $\mathrm{N} \times \mathrm{N}$ patch around each pixel is extracted from a given image, and the model is trained on these patches and given class labels to correctly identify classes such as normal brain and tumour. The designed networks contain multiple convolutional, activation, pooling, and fully connected layers sequentially.
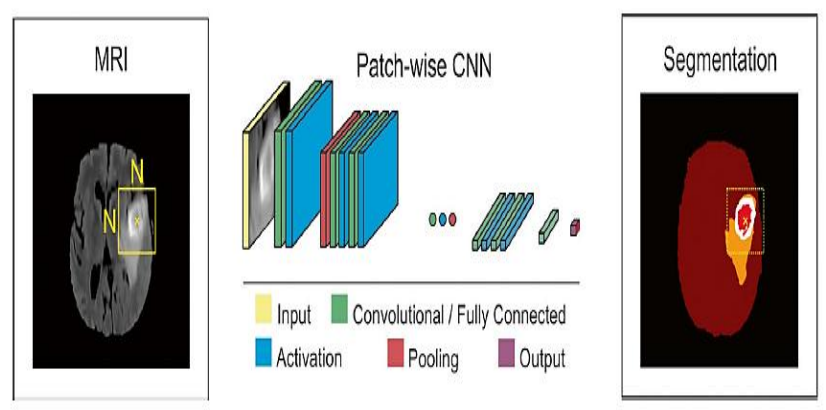

Fig. 1.5: Schematic illustration of a patch-wise CNN architecture for brain tumour segmentation task [15].

2. Semantic-Wise CNN Architecture: This type of architecture makes predictions for each pixel of the whole input image like semantic segmentation [5] [8]. Similar to auto encoders, they include encoder part that extracts features and decoder part that up samples or de-convolves the higher-level features from the encoder part and combines lower level features from the encoder part to classify pixels. The input image is mapped to the segmentation labels in a way that minimizes a loss function.
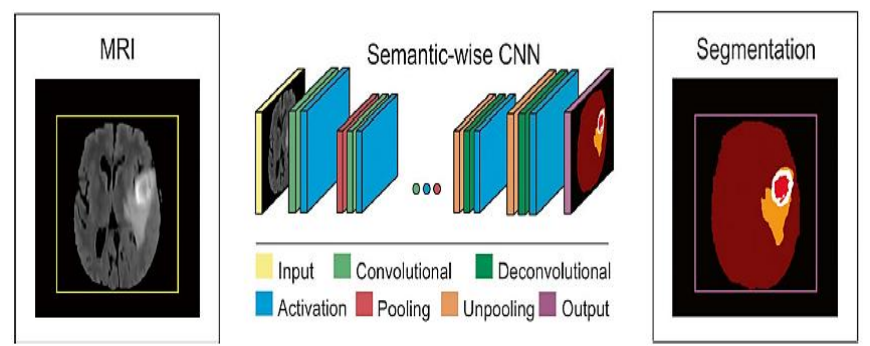

Fig. 1.6: Schematic illustration of asemantic-wise CNN architecture for brain tumour segmentation task

3. Cascaded CNN Architecture: This type of architecture combines two CNN architectures [6]. The output of the first $\mathrm{CNN}$ is used as an input to the second CNN to obtain classification results. is used to the model trained by the first
CNN with initial prediction of class labels while second CNN is used to further tune the results of the first CNN.

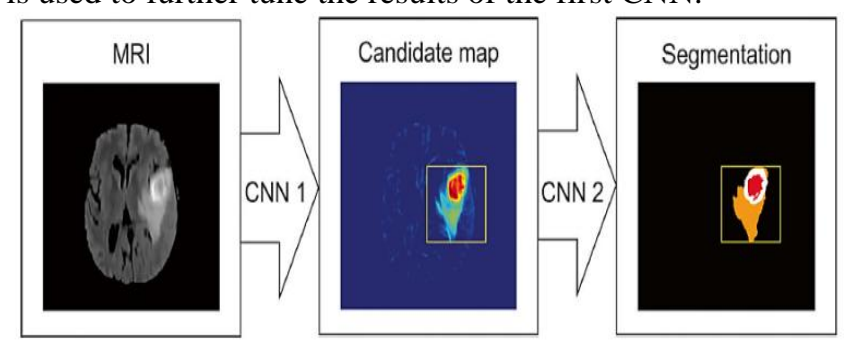

Fig. 1.7 Schematic illustration of a cascaded CNN architecture for brain tumour segmentation

where the output of the first network (CNN 1) is used in addition to image data for are fined input to the second network (CNN2), which provides final segmentation [15].

\section{RELATED WORK}

Bao, S. et.al [1] proposed a completely unique method for brain MR image segmentation has been with deep learning techniques so as to get preliminary labelling and graphical models to supply the ultimate result. a selected architecture, namely multi-scale structured convolutional neural networks (MS-CNN), is meant to capture discriminative features for every sub-cortical structure and to get a label probability map for the target image. Akkus, Z. et.al [2] aimed to supply a summary of current deep learning-based segmentation approaches for quantitative brain MRI. First, we review the present deep learning architectures used for the segmentation of cerebral brain structures and brain lesions. Next, the performance, speed, and characteristics of deep learning schemes are summarized and discussed. Finally, we offer a critical assessment of the present state and identify likely future developments and trends. Havaei, M. et.al [3] presented a method which was totally automatic brain tumour segmentation method based on Deep Neural Networks (DNNs). The proposed networks were tailored to Glioblastoma Multiforme (GBM) (both low and high grade) pictured in MR images. By their very nature, these tumours can appear anywhere within the brain and have almost any quite shape, size, and contrast. These reasons motivate our exploration of a machine learning solution that exploits a versatile, high capacity DNN while being extremely efficient. Akkus, Z. et.al [4] predicted the 1p/19q status from magnetic resonance imaging images using convolutional neural networks (CNN), which might be a non-invasive alternative to surgical biopsy and histopathological analysis. Proposed Method: Our method consists of three main steps: registration of image, tumour segmentation, and classification of $1 p / 19 q$ status using CNN. The Researchers included a total of 159 LGG(Low-Garde Gliomas) with 3 image slices each who had biopsy-proven 1p/19q status (Fifty Seven (57) non-deleted 
and One hundred two(102) co-deleted) and preoperative postcontrast- $\mathrm{T}_{1}\left(\mathrm{~T}_{1} \mathrm{C}\right)$ and $\mathrm{T}_{2}$ images. The experts divided our data into training, validation, and test sets. Firstly, the training data was balanced for equivalent class likelihood and was then improved with iterations of random translational shift, rotation, and horizontal and vertical flips to extend the dimensions of the training set. Finally, the analysts evaluated several configurations of a multi-scale $\mathrm{CNN}$ architecture until training and validation accuracies became consistent. Tom Brosch, et.al [5] proposed a novel segmentation deep 3D convolutional encoder networks supported scheme with shortcut connections and apply it to the segmentation of MS (MS) lesions in resonance images. The model consists of a neural network that consists of two interconnected pathways, a convolutional pathway, which learns increasingly more abstract and higher-level image features, and a deconvolutional pathway, which predicts the ultimate segmentation at the voxel level. A team of researchers have assessed our method on two publicly available data sets (MICCAI 2008 and ISBI 2015 challenges) with the results showing that our method performs comparably to the topranked state-of-the-art methods, even when only comparatively small data sets are available for training. Dou,Q. et.al [6] proposed a completely unique automatic method to detect CMBs from resonance (MR) images by exploiting the 3D convolutional neural network (CNN). Comparison with previous methods that employed either lowlevel hand-crafted descriptors or 2D CNNs, our method can take full advantage of spatial contextual information in MR volumes to extract more representative high-level features for CMBs, and hence achieve a way better detection accuracy. Moeskops, P. et.al [7] presented a way for the automated segmentation of MRI brain images into a variety of tissue classes implementing a convolutional neural network to ensure that the tactic obtains correct segmentation details also as spatial consistency, the network uses several patch sizes and several convolution kernel sizes to collect multi-scale information about each voxel. the tactic isn't hooked into explicit features but learns to recognize the knowledge that's important for the classification supported training data. the tactic requires one anatomical MR image only. Authors Nie, D. et.al [8] in this research for multimodality information content from T1, T2, and FA images separately the author particularly conducted a convolutional pooling stream and then combine them in high-layer for finally generating the segmentation maps because of the outputs. We compared the performance of our approach thereupon of the commonly used segmentation methods on a group of manually segmented isointense phase brain images. Results showed that our proposed model significantly outperformed previous methods in terms of accuracy. Additionally, our results also indicated a far better way of integrating multi-modality images, which results in performance improvement. Xavier Then Fernandez, T. et.al [9] proposed a substitute that achieves lesion and brain tissue segmentation through simultaneous estimation of a spatially global within-the-subject intensity distribution and a spatially local intensity distribution derived from a healthy reference population. The experts have demonstrated that MS lesions are often segmented as outliers from this intensity model of population and subject. They administered extensive experiments with both synthetic and clinical data and compared the performance of our new algorithm to those of state-of-the-art techniques.

\section{THE PROPOSED METHOD}

\subsection{Proposed Framework}

\subsubsection{Convolution}

Considering a network with $\mathrm{L}^{\sim} \geq 1$. Among L $\mathrm{L}^{\sim}$ layers, $\mathrm{L}^{\sim}-1$ represents the hidden type of layers. Let y_o represent the network input. For each of the layer $\tilde{I} \in\{\overline{1}, 2, \ldots, \tilde{L}\}$ set a_ $\tilde{1}$ $=\omega \_\tilde{l} \mathrm{~s}\left(\mathrm{a} \_(\tilde{\mathrm{l}}-1)\right)$ where $\mathrm{s}$ presents a vector-based function, $\mathrm{s}\left(\mathrm{a} \_0\right)=\mathrm{x}_{-} \mathbf{0}$. The layers of consecutive nature are interlinked. Let $\mathrm{f}\left(\omega, \mathrm{x} \_\overline{0}\right)$ and $\omega=\left(\omega_{-} \tilde{1}\right) \_\tilde{1}$ be the network output at end of $\mathrm{L}^{\mathrm{t}}$ th layers.

1. Local gradient-based back propagated error: Each of the layer $\tilde{I}$ involves various units. The local gradient-based back propagated error is usually defined by $(\mathrm{El}) \_\left(\mathrm{a} \_\mathrm{i}\right)$ as the partial derivative at $i^{\text {th }}$ unit: The use of rule-based on classical chain results in:

$(E l) \_\left(a \_i\right)=(E l) \_\left(a \_j\right) .\left(a \_j\right) \_w i j$

2. Linear Networks: We usually refer to the network of linear form when there is "s" type of mapping which identifies the function; $s(a)=a$ : In such type of case, the $f\left(\omega, x \_0\right)$ output function represents a weight-based polynomial function.

3. Maxout: This layer represents a simple layer where the activation-based function is the maxima of inputs.

4. Maxpooling: It is usually done by putting into use a maximized filter to sub-regions (non-overlapping) of the primary representation as shown in figure 3.1 below.

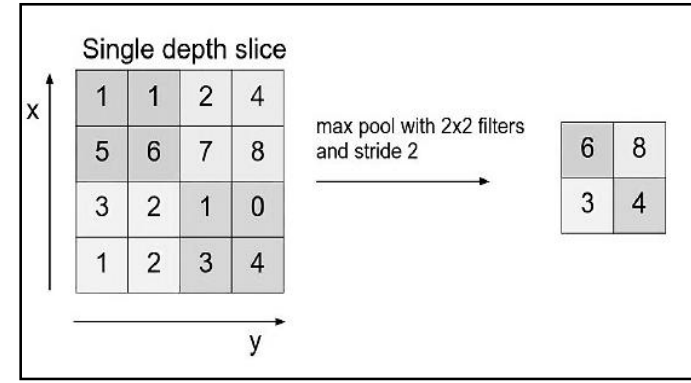

Fig. 3.1: Maxpool operation example

A unit of max-pooling ' $j$ ' outputs the maxima of all the unit outputs from where it accepts the inputs. Further to the process of max pooling, the units of pooling can perform 
various other types of functions like L2-norm pooling or even the process of average pooling.

5. Rectifiers: This represents a neuron layer that is applies the activation function of non-saturating form $s(a)=\max (0 ; a)$ : The other type of functions are mainly used for increasing the nonlinearity, for instance, the saturating form of hyperbolic tangent $s(a)=\tanh (a) ; s(a)=j \tanh (a)$ as the sigmoid function: The RLU i.e. Rectified Linear Units are mainly used in various kind of implementations.

6. Dropout: The technique of dropout helps in improving the neural networks and aims to mitigate or reduce the overfitting problem. It mainly comprises of dropping out all the units (visible and hidden) in the methodology of neural networks. With this technology, it usually ignores all the operations of that specific units, along with its outgoing and incoming links or connections.

7. Drop connect: It represents dropout refinement where instead of units, the links are dropped during the period of training.

8. Convolution layers: In a convolutional layer, the units of the convolutional layer shares weight through a discrete type of convolution.

\subsubsection{Learning the Network}

Neural network learning presents a supervised (controlled) method of classification using a set of data as an unlabeled or unmanaged object as an investment (input). Data collection is mainly divided into three of the following parts, known as Authentication Kit, Test Kit, and Training Collection. The set of training is mainly used to prepare or train the network in the periods referred to as epochs/eras, during this process the loss function calculates two significant values i.e. accuracy and loss. These values show the accuracy and error created by the network. The network for improving the design creates a correct mapping of output/ input, even in case if the input is little distinct from the instances used in the phase of training. If the system network is well-trained, we are risking too much adaptation of the training collection data as the network learns the database noise present in it. This phenomenon is known as over-fitting. The over-trained (prevailing) network is extremely tough and therefore loses its extensions. To prevent this issue, the so-called "early termination/stopping" method is used. Learning the set of training is usually carried out until the era when the value of the loss for the set of validation begins to boost, the moment over which the over fitting effect begins . After completion of the learning phase, the capability of classification of the network is further evaluated based on the operational cost of the loss calculated for the test package.

\subsection{Proposed methodology: Steps}

Step1: Input Brain MRI images.

Step 2: Pre-processed the image and denoise it.

Step 3: Next step is to extract the group of the same area.

Step 4: Apply the convolution process.
Step 4: After Convolution extracts the low-level features and grouped them.

Step 5: Then check the output if it is optimized then jumps to step 6 otherwise go to step 4 .

Step 6: Find the non-overlapping features and then analyze PSNR, MSE, and Accuracy.

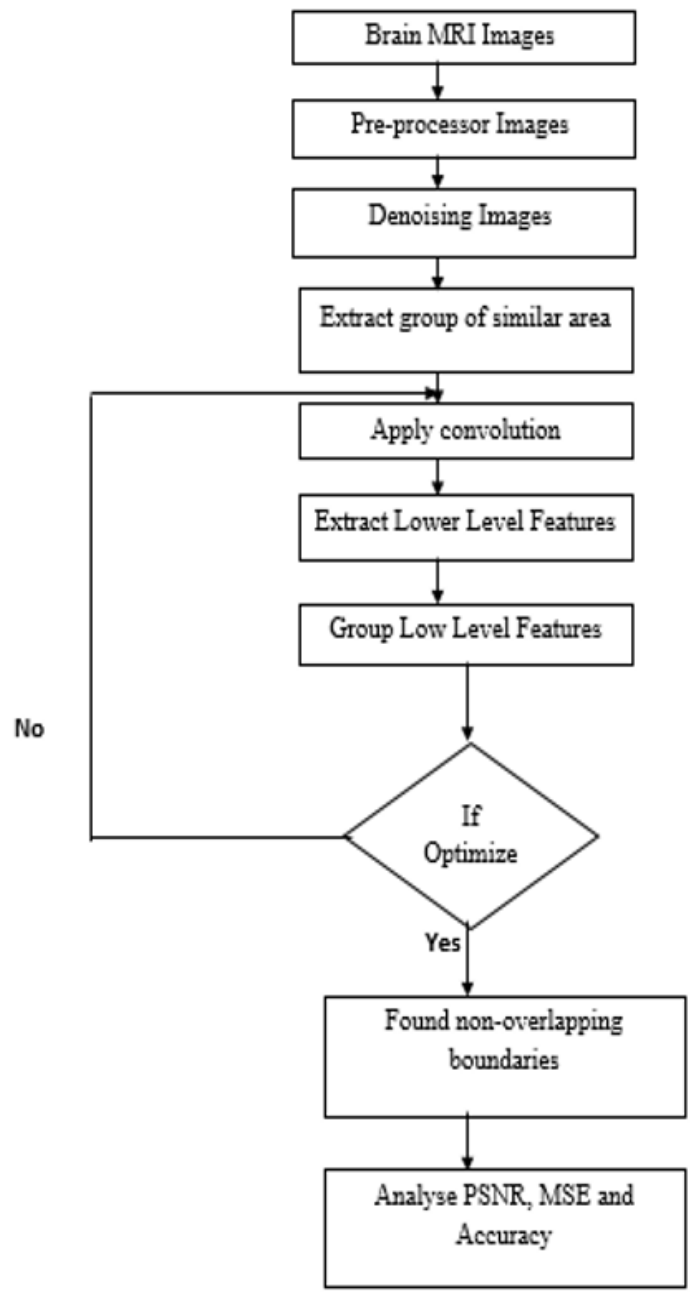

Fig. 3.2: Proposed Flowchart

\subsection{Algorithm Used}

1. Particle Swarm Optimization (PSO): is an optimization technique that is based upon bird flocking and fish schooling. Swarm is the collection of particles. There is some objective function whose value has to be optimized with PSO. The optimized value of the objective function will be some point in the search space. Every particle moves in the search space to find the point at which objective function is optimized. At any point of time, every particle has some position and velocity in the search space. Initially, positions and velocities of particles are randomly assigned. After each iteration, positions and velocities of particles are updated using 
equations 1 and 2. Each particle in PSO has its local best position and the global best position of the swarm. The optimal closer value is known as Global best position of the swarm. All the particles will move towards the global best position as it is close to the optimal value.

$\mathrm{V}_{\mathrm{i}, \mathrm{d}}(\mathrm{t}+1)=\alpha(\mathrm{t}) \mathrm{V}_{\mathrm{i}, \mathrm{d}}(\mathrm{t})+\beta_{\mathrm{p}} \operatorname{ran}_{\mathrm{p}}(\mathrm{t})\left(\right.$ persbest $\left._{\mathrm{i}, \mathrm{d}}-\mathrm{P}_{\mathrm{i}, \mathrm{d}}(\mathrm{t})\right)+$

$$
\beta_{\mathrm{g}} \text { ran }_{\mathrm{g}}(\mathrm{t})\left(\text { globest }_{\mathrm{d}}-\mathrm{P}_{\mathrm{i}, \mathrm{d}}(\mathrm{t})\right)
$$

$P_{i, d}(t+1)=P_{i, d}(t)+V_{i, d}(t)$

Where $\mathrm{Vi}, \mathrm{d}$ and $\mathrm{Pi}, \mathrm{d}$ is the velocity and position of particle I dimension $\mathrm{d}$ at iteration $\mathrm{t}+1 . \alpha(\mathrm{t})$ is the weight that tracks the history of velocity, $\beta_{\mathrm{p}}$ ranp $(\mathrm{t})$ and $\beta_{\mathrm{g}}$ rang $(\mathrm{t})$ are the random factors , persbest $t_{i, d}$ is the Personal Best of particle $I$ for dimension $\mathrm{d}$ and globest is the Global Best of the swarm for dimension $\mathrm{d}$.

\section{PSO}

Step 1: In PSO model for each particle i in $\mathrm{S}$ do

Step 2: for each dimension d in D do

Step 3: //initialize each particle's position and velocity

Step 4: $\quad \mathrm{x}_{\mathrm{i}, \mathrm{d}}=\operatorname{Rnd}\left(x_{\max }, x_{\min }\right)$

Step 5: $\quad v_{i, d}=R n d\left(-v_{\max } / 3, v_{\max } / 3\right)$

Step 6: end for

Step 7: //initialize particle's best position and velocity

$$
v_{i}(\mathrm{k}+1)=v_{i}(\mathrm{k})+\gamma 1_{\mathrm{i}}\left(p_{\mathrm{i}}-x_{\mathrm{i}}(\mathrm{k})\right)+\gamma_{2 i}\left(\mathrm{G}-x_{\mathrm{i}}(\mathrm{k})\right)
$$

New velocity

$$
x_{i}(\mathrm{k}+1)=x_{i}(\mathrm{k})+v_{i}(\mathrm{k}+1)
$$

Where

i- particle index

$\mathrm{k}$ - discrete time index

$v_{i}-$ velocity of $i^{\text {th }}$ particle

$\mathrm{x}_{\mathrm{i}}-$ position of $\mathrm{i}^{\text {th }}$ particle

$\mathrm{p}_{\mathrm{i}}$ - best position found by $\mathrm{i}^{\text {th }}$ particle(personal best)

G- best position found by swarm (global best, best of personal bests)

$\mathrm{G}_{(1,2) \mathrm{i}}$ - random number on the interval[0,1]applied to the $\mathrm{i}^{\text {th }}$ particle

Step 8: $p b_{i}=x_{i}$

Step 9: // update global best position

Step10: if $f\left(p b_{i}\right)<f(g b)$

Step 11: $g b=p b_{i}$

Step12: end if

Step13: end for

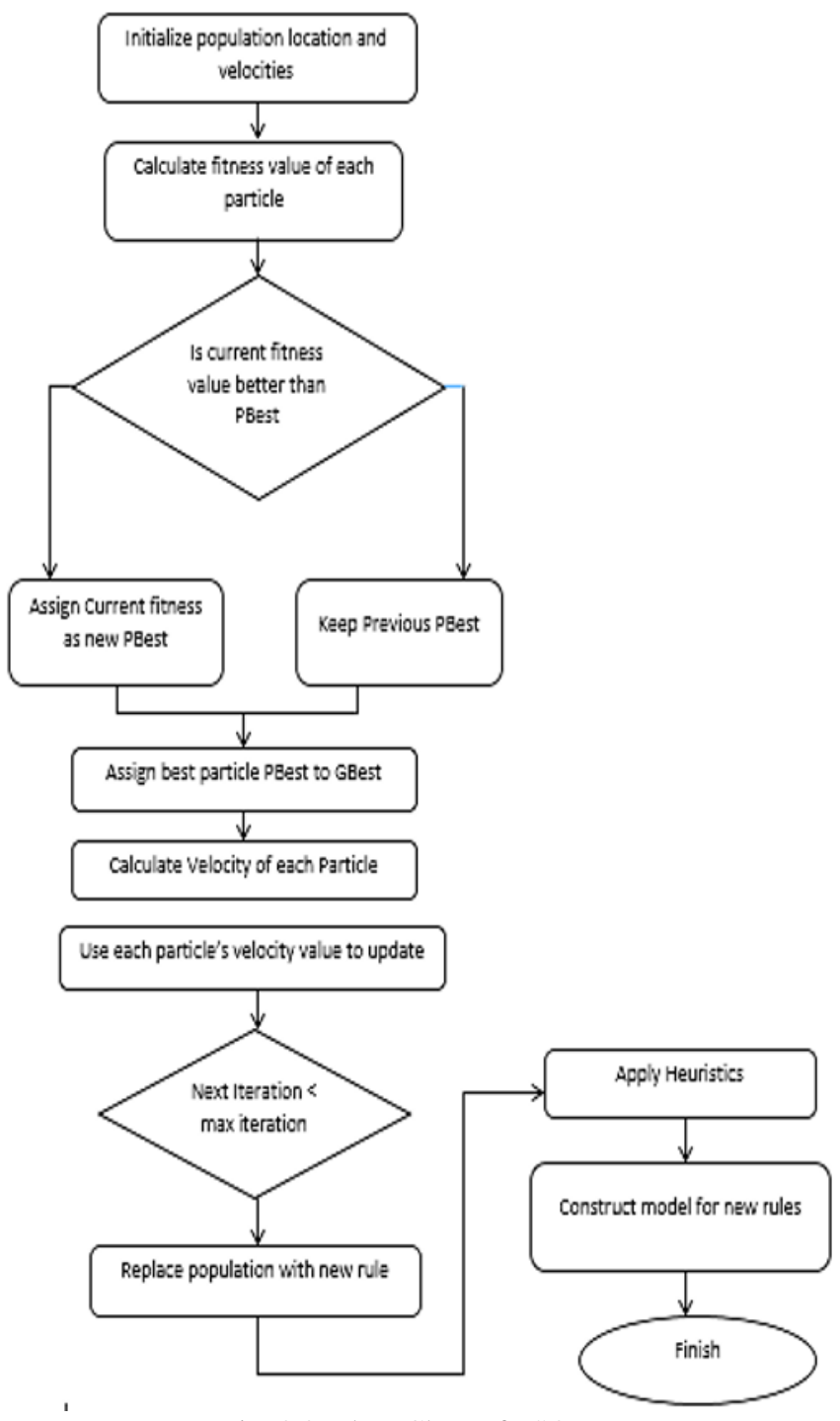

Fig. 3.3: Flow Chart of PSO

\section{RESULT ANALYSIS}

\subsection{Platform Used}

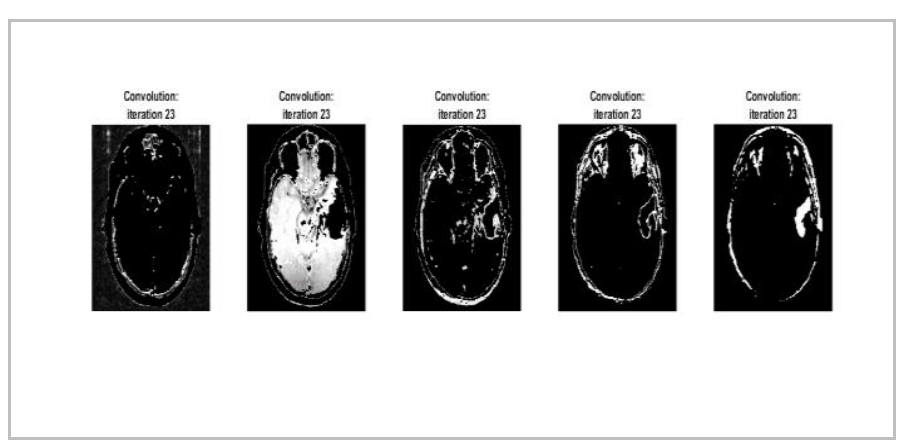

Fig.4.1: Different steps of image segmentation in Existing (convolution) approach 

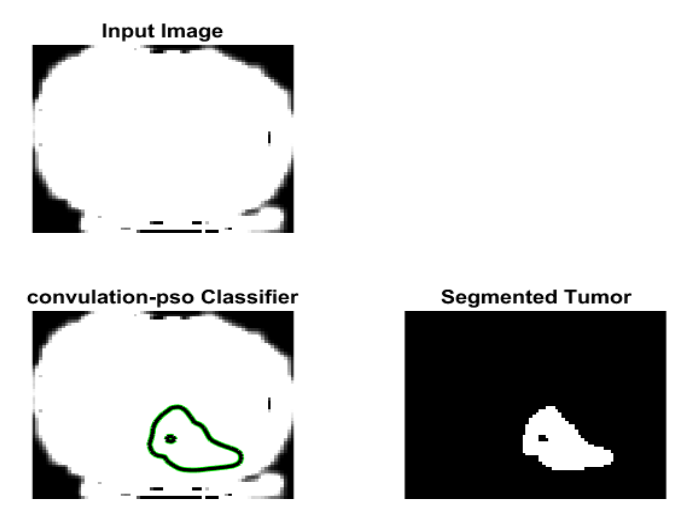

Fig. 4.2: Different steps of image segmentation in Existing (convolution) approach.

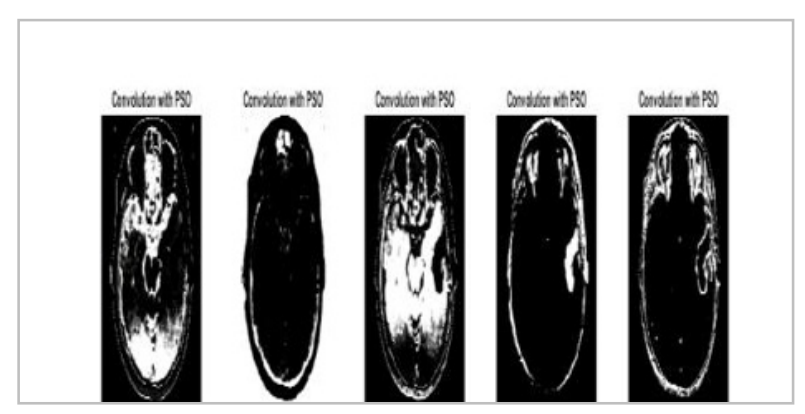

Fig. 4.3: Different steps of image segmentation in proposed (convolution-PSO) approach

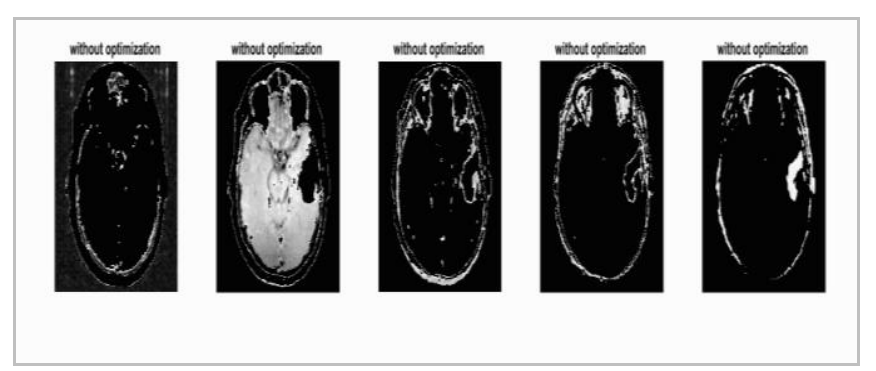

Fig. 4.4: Different steps of image segmentation in the Existing (without-optimization) approach
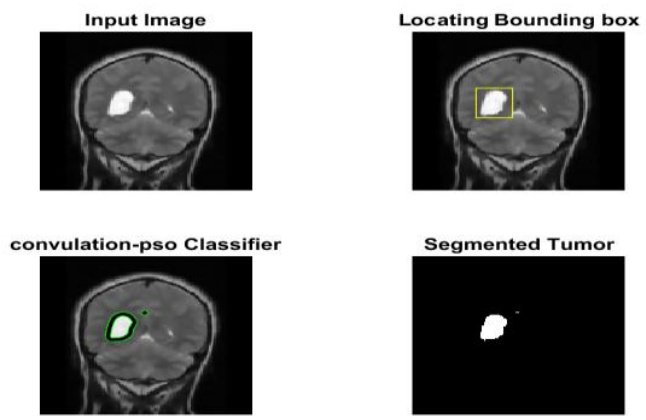

Fig.4.5: Different steps of image Classification in proposed (convolution-PSO) approach
Table 4.1: Comparison of PSNR between the existing and proposed approach

\begin{tabular}{|c|c|c|c|}
\hline Images & $\begin{array}{c}\text { PSNR } \\
\text { without } \\
\text { optimization }\end{array}$ & $\begin{array}{c}\text { PSNR- } \\
\text { Convolution }\end{array}$ & $\begin{array}{c}\text { PSNR- } \\
\text { Convolution- } \\
\text { PSO }\end{array}$ \\
\hline Image1 & 21.78 & 22.34 & 26.56 \\
\hline Image2 & 22.62 & 24.34 & 27.45 \\
\hline Image3 & 23.12 & 25.35 & 27.45 \\
\hline Image4 & 20.34 & 21.45 & 22.44 \\
\hline Image5 & 21.34 & 24.34 & 25.35 \\
\hline Image6 & 26.45 & 27.45 & 29.45 \\
\hline Image7 & 29.45 & 32.45 & 33.45 \\
\hline Image8 & 30.45 & 34.34 & 36.33 \\
\hline Image9 & 32.45 & 33.244 & 34.34 \\
\hline
\end{tabular}

Table 4.1 explains the comparison of PSNR between the proposed and the existing approaches. Here, PSNR represents the ratio between the maximized possible signal power and the power of corrupting noise that disturbs the reliability of its depiction. The value of PSNR (without optimization) has more corrupting noise and less signal power. But with PSNR (convolution), the value of corrupting noise reduces and the signal power is increased. Further, with PSNR (ConvolutionPSO), the signal power improves more and corrupting noise reduces further. For PSNR to be maximum, the corrupting noise should be less and the signal power should be more.

i.e. PSNR $=\frac{\text { Maxpossible signal power }}{\text { Power of corrupting noise }}$

For example, take the results of Image 1, the value gets improved for the case of convolution and it is further improved using the mechanism of Convolution-PSO approach.

PSNR (without optimization) $=21.78$

PSNR (Convolution) $=22.34$

PSNR (Convolution-PSO) $=26.56$

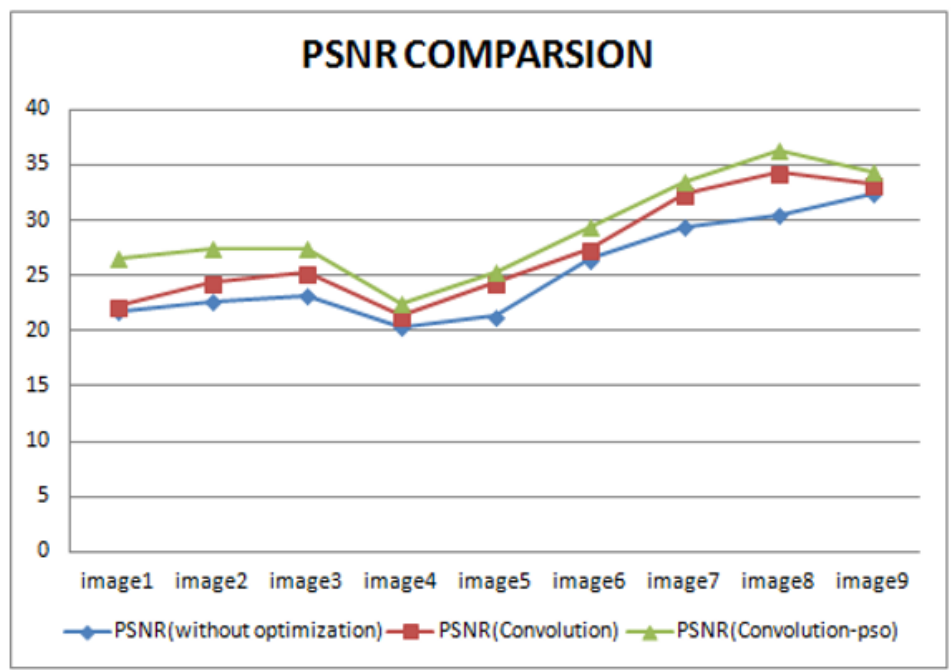

Fig. 4.6: Graphical representations of PSNR comparison between Proposed and Existing approaches. 
Figure 4.6 represents the graphical comparison of PSNR between the proposed and the existing approaches as per table 1 explained above.

Table 4.2: Comparison of Sensitivity between the existing and proposed approach

\begin{tabular}{|l|c|c|c|}
\hline Images & $\begin{array}{c}\text { Sensitivit } \\
\text { y without } \\
\text { optimizat } \\
\text { ion }\end{array}$ & $\begin{array}{c}\text { Sensitivity } \\
\text { Convolution }\end{array}$ & $\begin{array}{c}\text { Sensitivity } \\
\text { Convolution } \\
\text { PSO }\end{array}$ \\
\hline Image1 & 97.45 & 98.34 & 99.45 \\
\hline Image2 & 96.34 & 97 & 98.45 \\
\hline Image3 & 92.34 & 94.34 & 96.45 \\
\hline Image4 & 90.23 & 92.12 & 95.35 \\
\hline Image5 & 89.45 & 90.34 & 95.43 \\
\hline Image6 & 86.45 & 89.45 & 93.23 \\
\hline Image7 & 84.34 & 86.34 & 97.45 \\
\hline Image8 & 90.23 & 92.34 & 95.32 \\
\hline Image9 & 92.34 & 93.23 & 98.34 \\
\hline
\end{tabular}

Table 4.2 explains the comparison of Sensitivity between the proposed and the existing approaches. It represents the study that how the uncertainty in the output of a system can be distributed and assigned to diverse sources of uncertainty in its inputs. It is also called a true positive rate or the probability of detection. In terms of the medical field, it measures the actual positive or present proportion of a disease that are appropriately recognized. For instance, the sick people percentage who are appropriately recognized to have that particular condition of suffering. For example, take the results of Image 1, the value of sensitivity gets improved for the case of convolution and it is further improved using the mechanism of convolution-PSO approach. Same happens for other cases taken in the above mentioned table 4.2

Sensitivity (without optimization) $=97.45$

Sensitivity $($ Convolution $)=98.34$

Sensitivity (Convolution-PSO) $=99.45$

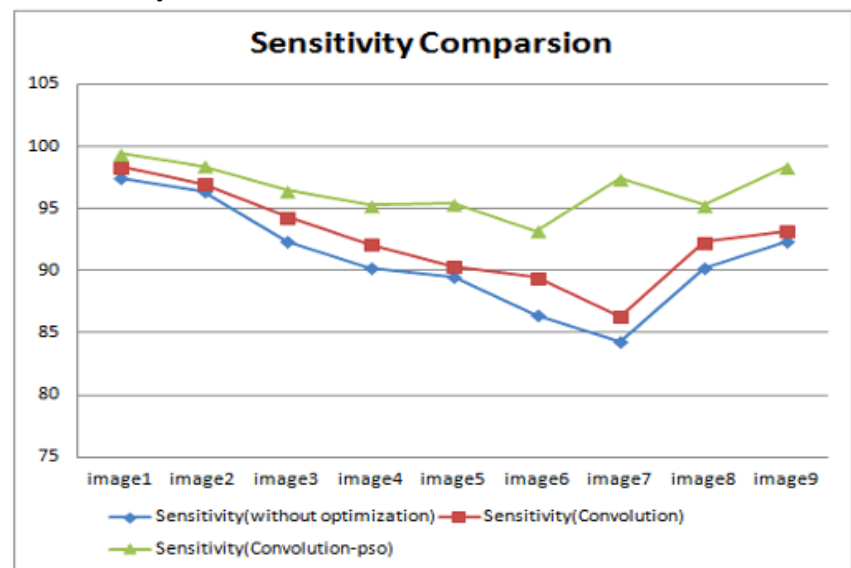

Fig. 4.7: Graphical representations of Sensitivity comparison between proposed and existing approaches.
Figure 4.7 represents the graphical comparison of sensitivity between the proposed and the existing approaches as per table 4.2 explained above.

Table 4.3: Comparison of Specificity between the existing and proposed approach

\begin{tabular}{|c|c|c|c|}
\hline Images & $\begin{array}{c}\text { Specificity } \\
\text { without } \\
\text { optimization }\end{array}$ & $\begin{array}{c}\text { Specificity } \\
\text { Convolution }\end{array}$ & $\begin{array}{c}\text { Specificity } \\
\text { Convolution } \\
\text { PSO }\end{array}$ \\
\hline Image1 & 96.895 & 97.67 & 98.95 \\
\hline Image2 & 94.34 & 95.67 & 97.45 \\
\hline Image3 & 91.285 & 93.23 & 95.9 \\
\hline Image4 & 89.84 & 91.23 & 95.39 \\
\hline Image5 & 87.95 & 89.895 & 94.33 \\
\hline Image6 & 85.395 & 87.895 & 95.34 \\
\hline Image7 & 87.285 & 89.34 & 96.385 \\
\hline Image8 & 91.285 & 92.785 & 96.83 \\
\hline Image9 & 92.34 & 93.23 & 98.34 \\
\hline
\end{tabular}

Table 4.3 explains the comparison of Specificity between the proposed and the existing approaches. It is also called a true negative rate or the probability of detection. In terms of medical field, it measure the actual negative or absent proportion of a disease that is appropriately recognized. For instance, the healthy people percentage who are appropriately recognized not to have that particular condition of suffering. For example, take the results of Image 1, the value of specificity gets improved for the case of convolution and it is further improved using the mechanism of convolution-PSO approach. Same happens for other cases taken in the abovementioned table 3

Specificity $($ without optimization $)=96.895$

Specificity (Convolution) $=97.67$

Specificity $($ Convolution-PSO $)=98.95$

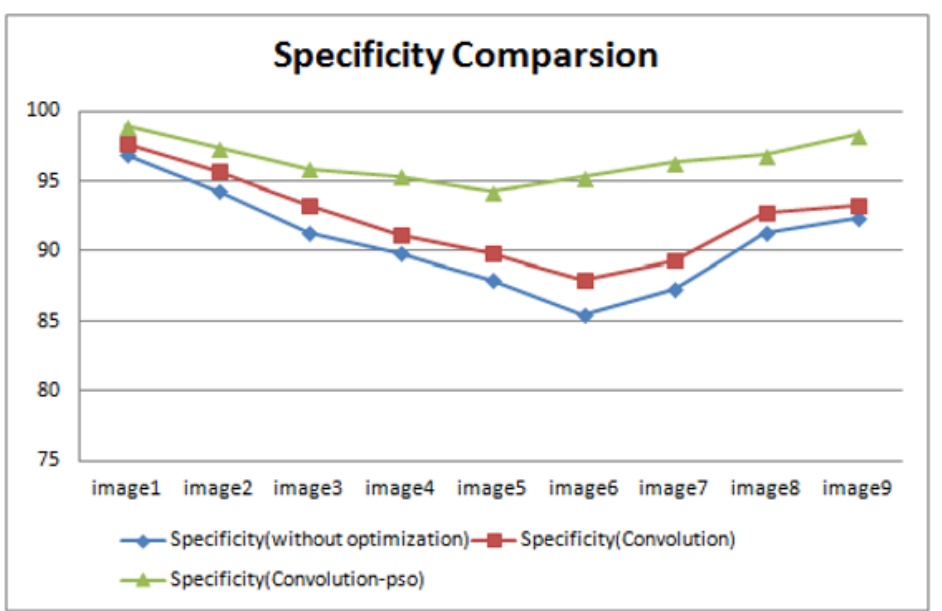

Fig. 4.8: Graphical representations of Specificity comparison between proposed and existing approaches. 
Figure 4.8 represents the graphical comparison of Specificity between the proposed and the existing approaches as per Table 4.3 explained above.

Table 4.4 Comparison of Accuracy between the existing and proposed approach

\begin{tabular}{|l|l|l|l|}
\hline Images & $\begin{array}{c}\text { Accuracy } \\
\text { without } \\
\text { optimization }\end{array}$ & $\begin{array}{c}\text { Accuracy } \\
\text { Convolution }\end{array}$ & $\begin{array}{c}\text { Accuracy } \\
\text { Convolution- } \\
\text { PSO }\end{array}$ \\
\hline Image1 & 94.1733333 & 95.5233333 & 97.4333333 \\
\hline Image2 & 91.8216666 & 93.3766666 & 96.2466666 \\
\hline Image3 & 89.6916666 & 91.4516666 & 95.2066666 \\
\hline Image4 & 87.7283333 & 89.6733333 & 95.02 \\
\hline Image5 & 86.8766666 & 89.0433333 & 95.3516666 \\
\hline Image6 & 87.9883333 & 90.0066666 & 96.185 \\
\hline Image7 & 90.3033333 & 91.785 & 97.185 \\
\hline Image8 & 91.8125 & 93.0075 & 97.585 \\
\hline Image9 & 92.34 & 93.23 & 98.34 \\
\hline
\end{tabular}

Table $4 . .4$ presents the depiction of systematic errors, and a quantity of arithmetic bias.

For example, take the results of Image 1, the value of accuracy gets improved for the case of convolution and it is further improved using the mechanism of convolution-PSO approach. Same happens for other cases taken in the abovementioned table 4.4

Accuracy (without optimization) $=94.17333333$

Accuracy $($ Convolution $)=95.52333333$

Accuracy (Convolution-PSO) $=97.43333333$

Figure 4.9 represents the graphical comparison of accuracy between the proposed and the existing approaches as per table 4.4 explained above.

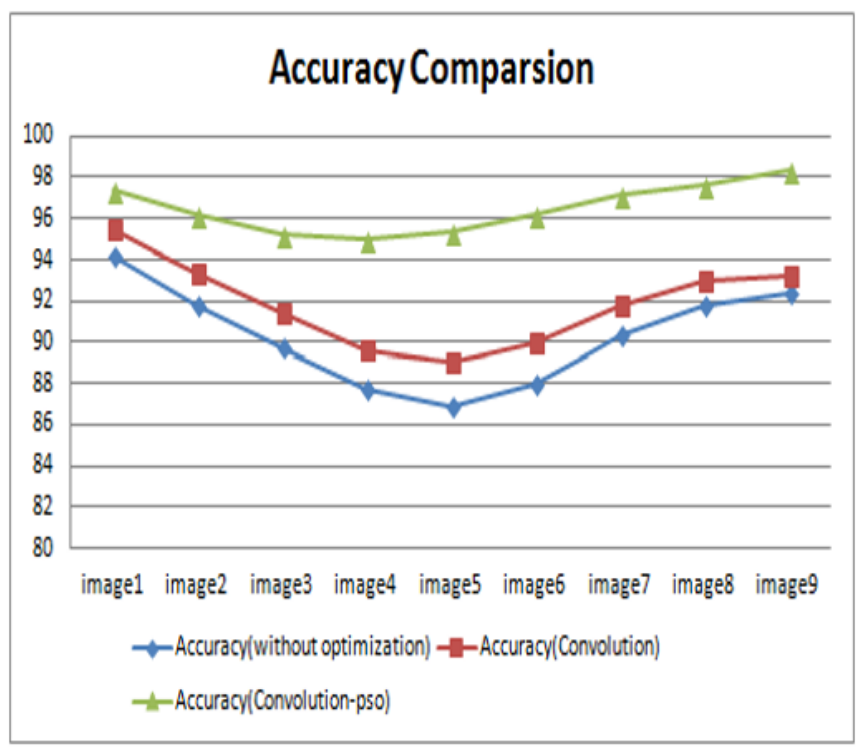

Fig. 4.9: Graphical representations of Accuracy comparison between proposed and existing approaches

\section{CONCLUSION}

In magnetic resonance imaging (MRI), Brain tumour segmentation is considered a multifaceted practice due to the variability of tumour shapes and the complexity of determining the tumour location, size, and texture. Manual tumour segmentation is a time-consuming task highly prone to human error. Hence, this study proposes an automated method that can identify tumour slices and segment the tumour across all image slices in volumetric MRI brain scans. First, a set of algorithms in the pre-processing stage is used to clean and standardize the collected data. Brain tumour segmentation algorithms have relatively good results in the field of medical image analysis, there is a certain distance in clinical applications. Due to a lack of interaction between researchers and clinicians, clinicians still rely on manual segmentation for a brain tumour in many cases. The existence of various tools aims to do pure research and is hardly useful for clinicians. Therefore, in the future, it will become inevitable to embed the developed tools into more user- friendly environments. Recently, some standard clinical acquisition protocols focusing on practicability studies are trying to formulate to improve the clinical applications more speedily. Apart from the evaluation of accuracy and validity for the results of brain tumour segmentation, computation time is also an important criterion. The current standard computation time is, in general a few minutes. The real-time segmentation will be hard to achieve, but computation time over a few minutes is unacceptable in clinical routine. Another crucial aspect for brain tumour segmentation methods is robustness. If an automatic segmentation technique fails in some cases, clinicians will lose their trust and not use this technique. Therefore, robustness is also one of the major assessment criteria for each new method applied in clinical practice. Some current brain tumour segmentation methods provide robust results within a reasonable computation time. In the proposed approach select the optimize block from the convolution process which improve the accuracy sensitivity, specificity and accuracy. For future scope It would be enhancing to explore the behaviour and output of the different form of neural networks like ANN, CNN, PNN, DNN and simple neural network by using a smaller number of labelled images to perform well. In future There is a need to introduce an automated expert system which can identify the tumour at its earlier stage so that better planning could be organized for treatment.

\section{REFERENCES}

[1] Bao, S., \& Chung, A. C. (2018), Multi-scale structured CNN with label consistency for brain MR image segmentation. Computer Methods in Biomechanics and Biomedical Engineering: Imaging \& Visualization, 6(1), 113117.

[2] Rubin, D.,Akkus, Z., Hoogi, A., Galimzianova, A., (2017), Deep learning for MRI brain segmentation: The state 
of the art and future directions. Journal of digital imaging, 30(4), 449-459.

[3] Havaei, M., Davy, A., Warde-Farley, D., Biard, A., Courville, A., Bengio, Y., \& Larochelle, H. (2017). Brain tumour segmentation with deep neural networks. Medical image analysis, 35, 18-31.

[4] Akkus, Z., Ali, I., Sedlář, J., Kline, T. L., Agrawal, J. P., \& Parney, I. F. Predicting 1p19q Chromosomal Deletion of LLG Low-Grade Gliomas from Medical Resonance Images Using Deep Learning.(2016).

[5] Brosch, T., Tang, L. Y., Yoo, Y., Li, D. K., Traboulsee, A. (2016),Deep 3D convolutional encoder networks with shortcuts for multiscale feature integration applied to multiple sclerosis lesion segmentation. IEEE transactions on medical imaging, 35(5), 1229-1239.

[6] Dou, Q., Chen, H., Yu, L., Zhao, L., Qin, J., Wang, D., \& Heng, P. A. (2016), Automatic detection of cerebral microbleeds from MR images via 3D convolutional neural networks. IEEE transactions on medical imaging, 35(5), 11821195.

[7]. Moeskops, P., Viergever, M. A., Mendrik, A. M., de Vries, L. S., Benders, M. J., \& Išgum, I. (2016). Automatic segmentation of Magnetic Resonance brain images with a convolutional neural network. IEEE transactions on medical imaging, 35(5), 1252-1261.

[8] Nie, D., Wang, L., Gao, Y., \& Sken, D. (2016, April), Fully convolutional networks for multi-modality isointense infant brain image segmentation. In 2016, IEEE 13th The International Symposium on Biomedical Imaging (ISBI) (pp. 1342-1345). IEEE.

[9] Tomas-Fernandez, X., \& Warfield, S. K. (2015), (MOPS) A model of population and subject intensities with application to multiple sclerosis lesion segmentation. IEEE transactions on medical imaging, 34(6), 1349-1361.

[10] Zhang, W., Li, R., Deng, H., Wang, L., Lin, W., Ji, S., \& Shen, D. (2015). Deep convolutional neural networks(DNN) for multi-modality isointense infant brain image segmentation. NeuroImage, 108, 214-224.

[11] Wang, L., Gao, Y., Shi, F., Li, G., Gilmore, J. H., Lin, W., \& Shen, D. (2015). LINKS: Learning based multi-source Integration framework for Segmentation of infant brain images. NeuroImage, 108, 160-172.

[12] Maier, O., Schröder, C., Forkert, N. D., Martinetz, T., \& Handels, H. (2015),Classifiers for ischemic stroke lesion segmentation: a comparison study. PloS one, 10(12), e0145118.

[13] De Brebisson, A., \& Montana, G. (2015). Deep neural networks for anatomical brain segmentation. In the proceedings of the IEEE Conference on Computer Vision and Pattern Recognition Workshops (pp. 20-28).

[14] Weiss, N., Rueckert, D., \& Rao, A. (2013, September), Multiple sclerosis lesion segmentation using dictionary learning and sparse coding. In International Conference on Medical Image Computing and Computer-Assisted Intervention (pp. 735-742). Springer, Berlin, Heidelberg.
[15] Senthilkumaran, N., \& Rajesh, R. (2011), Brain image segmentation. International journal of wisdom based computing, 1(3), 14-18.

[16] https://tbichallenge.wordpress.com/data

[17] https://portal.fli-iam.irisa.fr/msseg-challenge/data 